\title{
Guidewire Across Lesion
}

National Cancer Institute

\section{Source}

National Cancer Institute. Guidewire Across Lesion. NCI Thesaurus. Code C100082.

A measure of procedural success during a percutaneous coronary intervention when a guidewire can be deployed across a coronary artery lesion. (ACC) 